\title{
Laparoscopic management of colovesical fistula secondary to sigmoid diverticulitis: case report and the role of intraoperative indocyanine-green fluorescence
}

\author{
Emanuele Asti ${ }^{1}$, Daniele Bernardi ${ }^{1}$, Erika Andreatta $^{1}$, Andrea Conti ${ }^{2}$, Luca Carmignani ${ }^{2}$, Luigi Bonavina $^{1 \wedge}$ \\ ${ }^{1}$ Division of General Surgery, Department of Biomedical Sciences for Health, University of Milan, Milan, Italy; ${ }^{2}$ Division of Urology, IRCCS \\ Policlinico San Donato, San Donato Milanese, Italy \\ Correspondence to: Prof. Luigi Bonavina. Piazza Malan 1, IRCCS Policlinico San Donato, San Donato Milanese, 20097 Milan, Italy. \\ Email: luigi.bonavina@unimi.it.
}

\begin{abstract}
Colovesical fistula is characterized by multifactorial etiology and the diagnosis is often challenging. Sigmoid diverticulitis accounts for more than two-third of the cases. Pneumaturia and air in the bladder at computed tomography are the most typical clinical-radiologic signs. There is still no evidencebased consensus on the optimal surgical approach for this rare condition. Sigmoid resection with or without bladder resection/enteral stoma are the conventional therapeutic options, but ureter identification may be difficult during the procedure and injury from a direct or indirect trauma can occur. Therefore, double-J stenting is commonplace during open surgical procedures. The laparoscopic approach has been shown to be feasible and effective in case-series, but ureteral protection may be difficult because of the limited tactile feedback. We report the case of a 55-year-old male patient with colovesical fistula complicating repeated episodes of acute left colon diverticulitis and eventually treated with one-stage bladder-sparing laparoscopic sigmoid resection. Indocyanine-green (ICG) fluorescence was used intraoperatively for visualization of the left ureter and for assessment of colonic blood perfusion. The post-operative course was uneventful. Laparoscopy is feasible, safe, and effective for the treatment of colovesical fistula. Magnified vision enhanced by fluorescence imaging allows a precise surgical dissection and has the potential to decrease complication rates and to improve postoperative outcomes.
\end{abstract}

Keywords: Sigmoid diverticulitis; colovesical fistula; laparoscopic sigmoid resection; indocyanine-green fluorescence (ICG fluorescence); case report

Received: 25 June 2020; Accepted: 18 December 2020; Published: 20 January 2023.

doi: $10.21037 /$ jovs-20-140

View this article at: http://dx.doi.org/10.21037/jovs-20-140

\section{Introduction}

Colonic diverticular disease is widespread in Western countries and is often complicated by recurrent episodes of acute diverticulitis and perforation that may require emergency surgery and a Hartmann procedure. Colovesical fistula is a rare condition caused by sigmoid diverticulitis in approximately two-thirds of the patients. The incidence of colovesical fistula is estimated to be three times less in women because of the protective effect of the uterus interposed between the sigmoid colon and the bladder. Pneumaturia, fecaluria, and urinary tract infections are the most common features due to the high pressure gradient between the colonic lumen and the urinary tract system. Other symptoms and signs may include lower abdominal pain, fever, and peritonism. Diagnostic methods include

\footnotetext{
$\wedge$ ORCID: 0000-0002-4880-1670.
} 


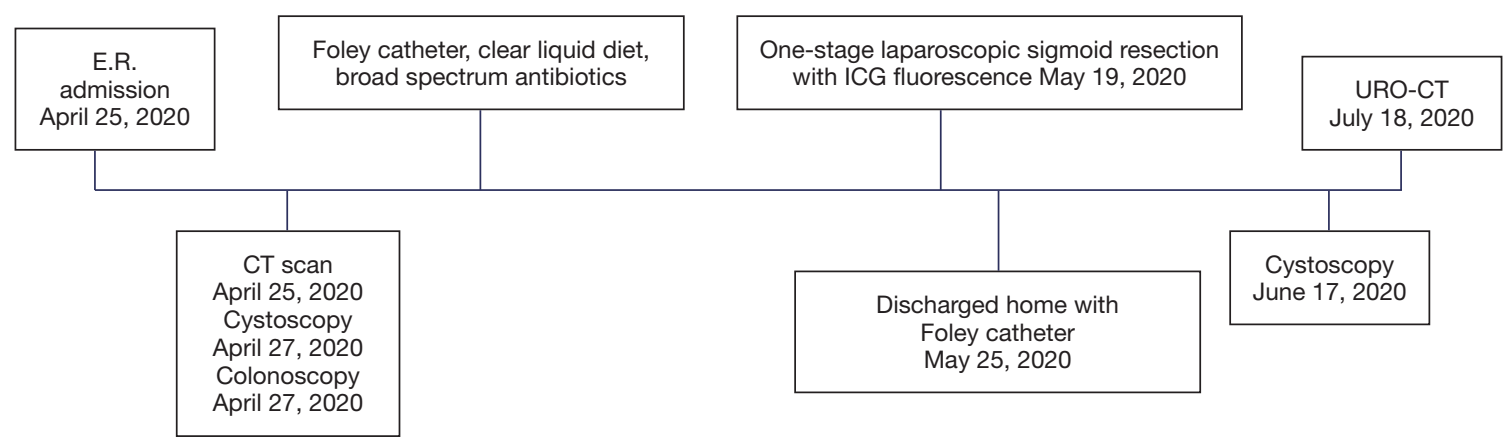

Figure 1 Timeline including work-up, patient preparation, laparoscopic management, and follow-up. E.R., emergency room; ICG, indocyanine-green.

abdomino-pelvic computed tomography (CT) scan, magnetic resonance (MR), cystoscopy and colonoscopy, all with a rather low sensitivity (1). Although conservative treatment is feasible in selected high-risk patients, surgical therapy offers the best chance of cure given the risk of bladder outlet obstruction and urosepsis that require repeated courses of antibiotics and urinary catheterization.

The laparoscopic approach has been reported to be feasible and effective, but conversion rate to an open procedure has been reported in up to $50 \%$ of patients depending on the degree of bladder infiltration (2). Traditionally, especially in open surgery, use of ureteral stents was common practice for intraoperative identification of the ureters. During laparoscopic pelvic surgery, ureteral injuries occur in up to $10 \%$ of patients and are often diagnosed post-operatively (3). Near-infrared fluorescence with indocyanine-green (ICG) injected intravenously is a technology most commonly used for the intraoperative assessment of biliary anatomy and bowel perfusion. Local ICG injection through a ureteral probe rapidly stains the urothelium, allows prompt visualization of the ureter and may reduce morbidity associated with ureteral stents. We present the following case in accordance with the CARE reporting checklist (available at https://jovs.amegroups. com/article/view/10.21037/jovs-20-140/rc).

\section{Case presentation}

The clinical course and timeline of diagnosis and treatment is summarized in Figure 1.

\section{Patient selection and workup}

A 55-year-old Caucasian male presented to the emergency room with acute lower abdominal pain and a 1-week history of refractory constipation. He did not report stranguria, pneumaturia, or fecaluria. Past medical history was unremarkable except for multiple recurrent episodes of acute sigmoid diverticulitis. Blood pressure was 130/78 $\mathrm{mmHg}$, heart rate $100 \mathrm{bpm}$, and oxygen saturation $97 \%$ on room air. Body temperature was normal. Physical exam showed mild abdominal distension and tenderness in the lower abdominal quadrants. Abdominal ultrasound showed a small fluid collection in the left lower quadrant. Blood tests showed increased C-reactive protein $(13.7 \mathrm{mg} / \mathrm{dL})$ and white blood count $(12,500 / \mu \mathrm{L}$ with $86 \%$ neutrophils). Plain abdominal $\mathrm{X}$-ray did not reveal free air nor air-fluid images. A CT scan showed a complicated distal left colon diverticulitis with a long segment $(7 \mathrm{~cm})$ wall thickening, luminal narrowing (Figure $2 A$ ), and multiple perivisceral abscesses (Figure 2B). Moreover, air bubbles inside the bladder were visible (Figure 3), raising the suspicion of a colovesical fistula (Figure 4A,4B). The patient underwent a cystoscopy, which confirmed the presence of a fistula on the posterior-lateral wall of the bladder $2 \mathrm{~cm}$ above the vesical trigone (Figure 5). A colonoscopy ruled out the presence of malignancy. The patient was treated with a Foley catheter, clear liquid diet, and broad spectrum antibiotics. Blood tests normalized in a few days, and the patient was discharged home. Readmission for surgical treatment was planned in 3 weeks.

\section{Pre-operative preparation}

The patient maintained the urinary catheter for 4 weeks to reduce intra-bladder pressure and to allow spontaneous healing of the fistula. Low-residue diet was allowed. Oral bowel preparation with polyethylene glycol was administered 24 hours before surgery. Cefazoline $2 \mathrm{~g}$ and 

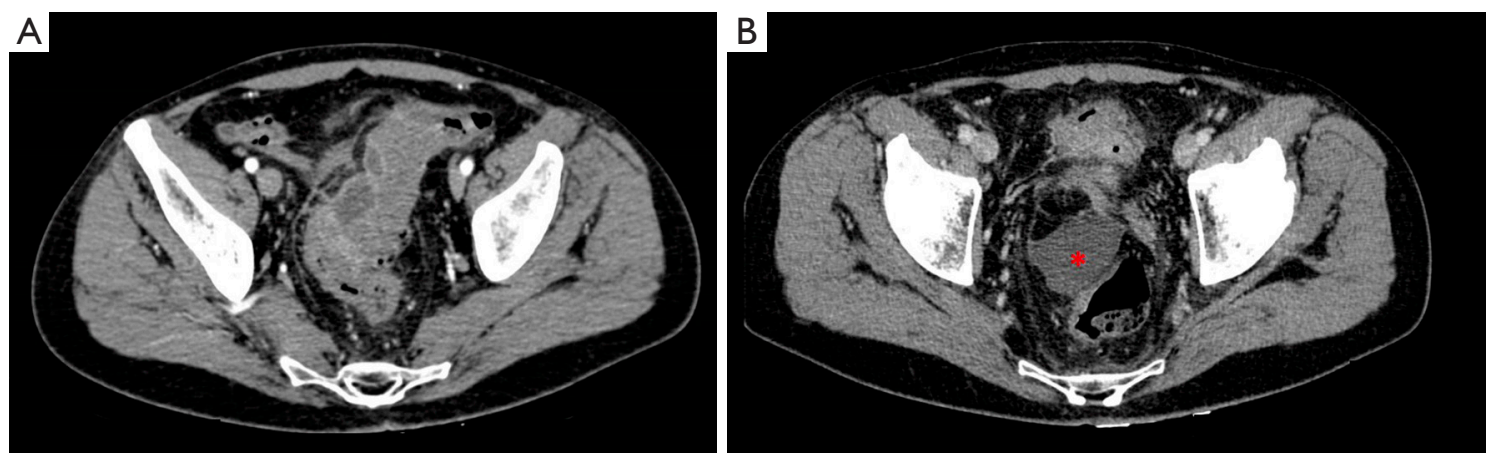

Figure 2 CT scan showing distal left colon wall thickening and luminal narrowing (A), and pelvic collection (red mark) (B).

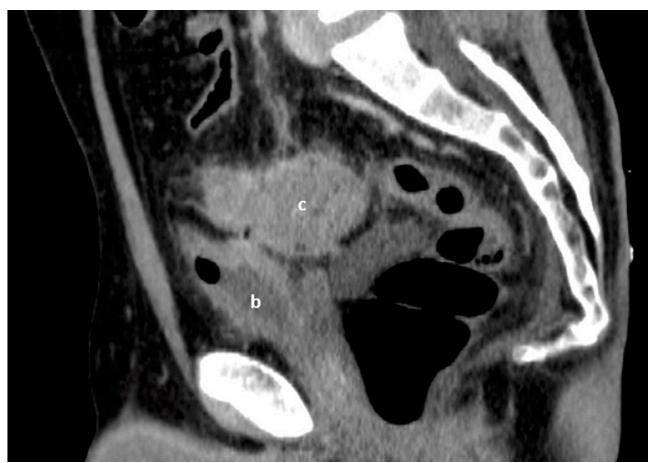

Figure 3 Air bubbles are visible inside the bladder (b), strictly adherent to the colonic wall (c).

metronidazole $500 \mathrm{mg}$ were given as antibiotic prophylaxis.

\section{Equipment preference cart}

* Visera Elite II OTV-S300 ${ }^{\circledR}$ video system (Olympus, Shinjuku, Tokyo, Japan);

* Visera Elite II CLV-S200-IR ${ }^{\circledR}$ light source (Olympus, Shinjuku, Tokyo, Japan);

* 12-mm and 5-mm laparoscopic trocar;

* 10-mm 30 laparoscope;

* Laparoscopic Croce-Olmi grasper (Karl Storz, Tuttlingen, Germany);

* Laparoscopic single-use Endo Clinch (Covidien, Dublin, Ireland);

* Laparoscopic peanuts;

* Laparoscopic Endopath Electrosurgery Probe Plus II ${ }^{\circledR}$ (Ethicon, Somerville, New Jersey, USA);

* Laparoscopic Ultracision Harmonic Scalpel ${ }^{\circledR}$ (Ethicon, Somerville, New Jersey, USA);

* Hem-o-lok clip (Weck Closure Systems, Teleflex,
Wayne, Pennsylvania, USA);

* Contour $^{\circledR}$ Stapler (Ethicon, Somerville, New Jersey, USA);

* Echelon ${ }^{\circledR}$ circular powered stapler 29 mm (Ethicon, Somerville, New Jersey, USA);

- $\quad$ Alexis $^{\circledR}$ wound retractor (Applied Medical, Rancho Santa Margarita, CA, USA);

- ICG Verdye ${ }^{\circledR} 5$ mg/mL (Diagnostic Green GmbH, Aschheim, Germany).

\section{Procedure}

All procedures performed in this study were in accordance with the ethical standards of the institutional research committee and with the Helsinki Declaration (as revised in 2013). Written informed consent was obtained from the patient for publication of this case report and accompanying images and videos. A copy of the written consent is available for review by the editorial office of this journal.

The patient was positioned supine on the operative table with the legs abducted. Under general anesthesia, a cystoscopy was performed, and a 6 Fr Pollack catheter was introduced in the left ureter to allow intraoperative injection of ICG. Pneumoperitoneum was induced through a Veress needle placed in the umbilicus. Four ports (two $12-\mathrm{mm}$ and two $5-\mathrm{mm}$ ) were placed as for laparoscopic left colectomy (Figure 6). Upon exploration of the abdominal cavity, the sigmoid was tenaciously adherent to the posterior-lateral aspect of the bladder (Figure 7). The small bowel was retracted away from the operative field. Partial mobilization of the descending and sigmoid colon was performed using the monopolar hook and harmonic scalpel. The inferior mesenteric artery and vein were clipped with Hem-o-lok and sectioned (Figure 8). The left ureter was visually identified and followed along its route 

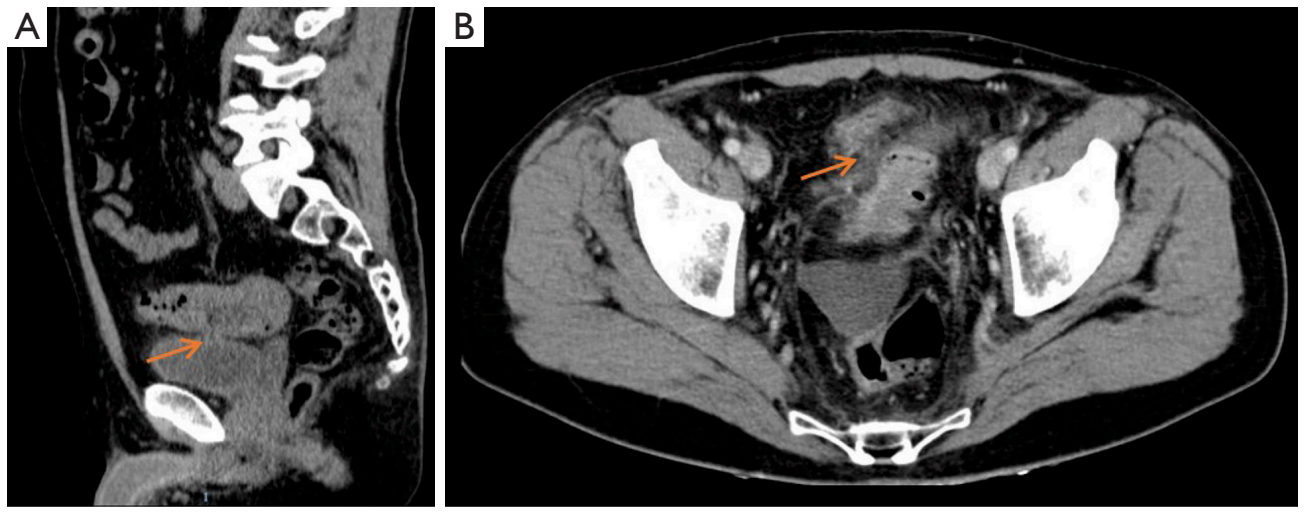

Figure 4 CT scan showing colovesical fistula (arrow) on sagittal (A) and axial plane (B).

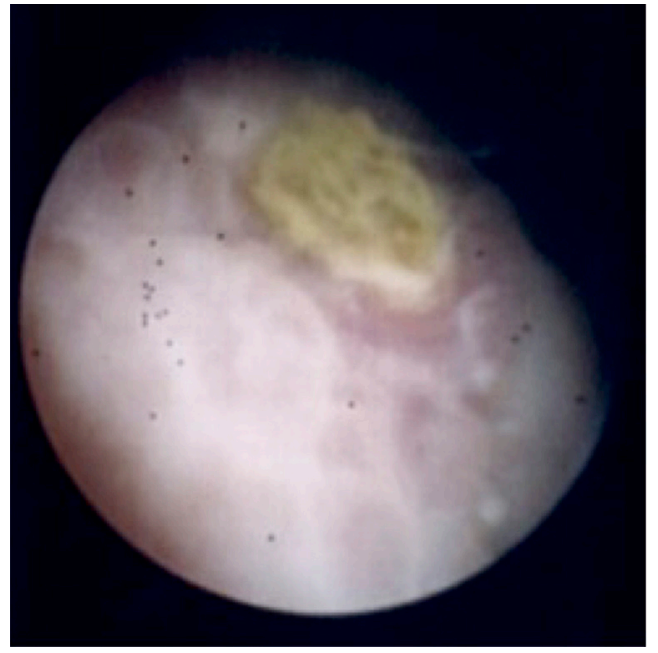

Figure 5 Cystoscopy showing fecal leakage from the left posterior wall of the bladder.

toward the pelvis, then it could not be clearly distinguished from the pseudotumor involving the sigmoid colon and the bladder. Infusion of $5 \mathrm{~mL}$ of ICG through the ureteral catheter allowed to visualize the left ureter (Figure 9) and guided the dissection of the sigmoid from the bladder wall. At the end of dissection, the bladder wall appeared undamaged, and a methylene blue test was performed to exclude urinary leakage (Figure 10). A 5-cm laparotomy in the right iliac fossa was performed and the Alexis ${ }^{\circledR}$ wound retractor was placed. A curved linear stapler (Contour ${ }^{\circledR}$ ) was introduced and sealed with a surgical glove to maintain the pneumoperitoneum. After rectal stapling, a $5 \mathrm{~mL}$ intravenous bolus of ICG was injected to test for colonic perfusion and to identify the ideal site for the anastomosis

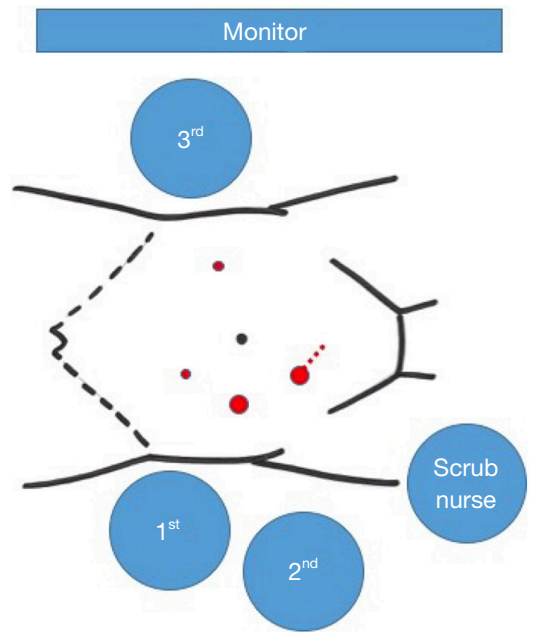

Figure 6 Laparoscopic setting and trocar positioning.

on the descending colon. A standard transanal KnightGriffen colorectal anastomosis was performed using a 29-mm circular stapler. Two abdominal drains were left in the pelvis, and the abdominal incisions were closed. Total duration of the procedure was 220 minutes, and blood loss was $<100 \mathrm{~mL}$. Video 1 shows the whole surgical procedure.

\section{Role of team members}

The surgical team consisted of two staff general surgeons (first operator and camera holder) on the right patient's side and one general surgery resident on the left patient's side (traction and support). The anesthesist, the surgical scrub nurse, the anesthesist nurse, and one auxiliary nurse 


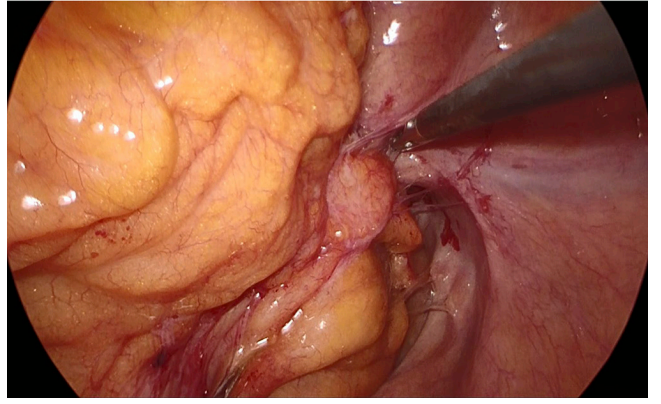

Figure 7 Laparoscopic view of the inflammatory pseudotumor surrounding the colovesical fistula.

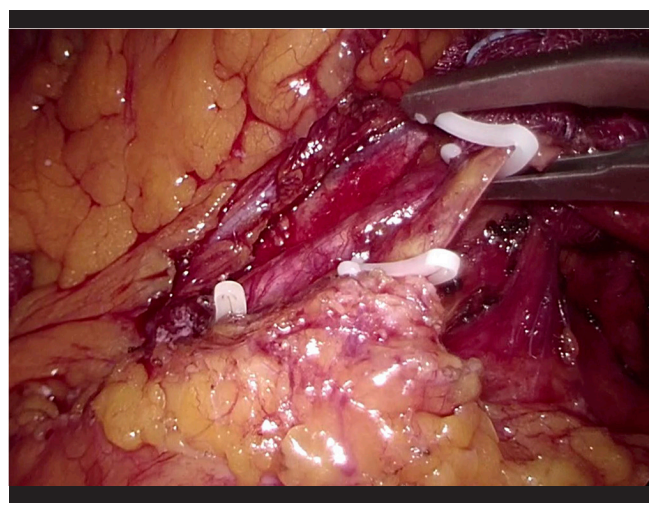

Figure 8 Dissection of the inferior mesenteric artery using Hemo-lok clips.

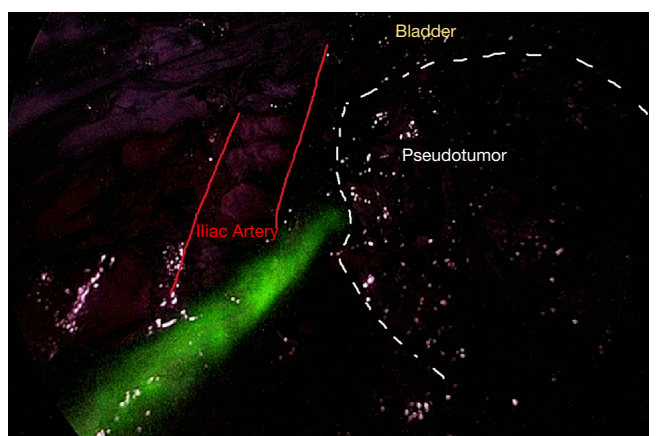

Figure 9 Use of ICG-fluorescence to identify the left ureter. ICG, indocyanine-green.

were part of the operating room staff. The consultant urologists who performed the preoperative cystoscopy were involved in the ureter cannulation and remained on call to provide assistance in case a partial cystectomy could

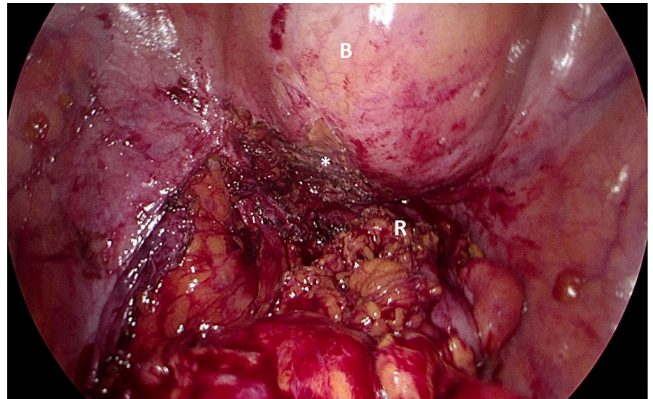

Figure 10 Laparoscopic view of the pelvis after dissection of the colovesical fistula $\left(^{*}\right)$. The bladder (B) is filled with methylene blue solution, and there is no evidence of leaks. $\mathrm{R}$, rectal stump.

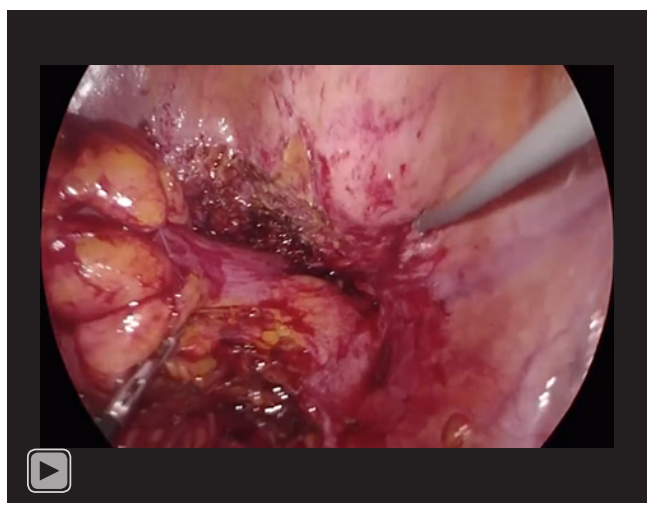

Video 1 Laparoscopic treatment of colovesical fistula in a patient with sigmoid diverticulitis.

have become necessary.

\section{Post-operative management}

The ureteral catheter was removed at the end of the procedure. The postoperative course was uneventful. Liquid diet was initiated on the second postoperative day. The patient was discharged on the $6^{\text {th }}$ postoperative day with a Foley catheter in place. An outpatient cystoscopy was performed three weeks later by the consultant urologist to confirm the complete healing of the bladder, and the urinary catheter was removed. Histopathology examination of the surgical specimen confirmed the inflammatory nature of the pseudotumor. At the 3-month follow-up the patient reported normal urinary and bowel function, was very pleased with the outcome of the procedure and returned to his usual business activity. A URO-CT scan showed normal 

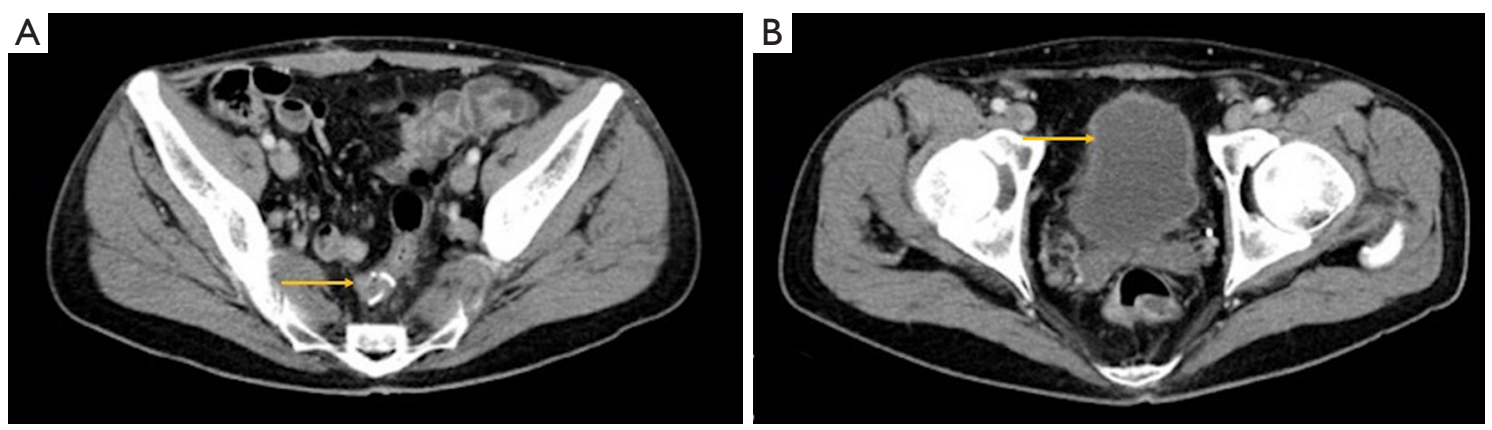

Figure 11 Postoperative CT scan showing normal findings. Arrows indicate the mechanical colorectal anastomosis (A) and the bladder without air-fluid levels (B).

findings (Figure 11).

\section{Tips, tricks, and pitfalls}

* An integrated surgical and urological team is a prerequisite for a successful outcome of the procedure.

* In case of acute diverticulitis (Hinchey 1-2), leave an indwelling urinary catheter and proceed with broad spectrum antibiotic therapy for at least 3 weeks before surgery to facilitate recovery from inflammation/ infection and spontaneous fistula healing.

* Laparoscopy may allow a bladder-sparing, one-stage minimally invasive sigmoid resection.

* Bladder leak testing with methylene blue should be routinely performed intraoperatively.

* ICG fluorescence through a ureteral catheter helps to visualize the ureter and may reduce the risk of intraoperative injuries.

* Intravenous ICG is useful to visualize colonic perfusion after rectal stapling and to choose the ideal site for the colorectal anastomosis.

\section{Discussion}

This case study is exemplary of the ideal, bladder-sparing management of a benign colovesical fistula, and adheres to the CARE guidelines (4). Our patient had a prior history of recurrent episodes of sigmoid diverticulitis, and presented with acute abdominal pain and no urological symptoms/ signs. The diagnosis of colovesical fistula was suspected on CT scan and subsequently confirmed by cystoscopy.

Laparoscopic colorectal resection for sigmoid diverticulitis has been shown to reduce postoperative morbidity and to increase quality of life compared to the open approach in a randomized trial (5). Although formal comparative studies of surgical therapy in colovesical fistula are lacking, the laparoscopic approach has been shown to be safe and effective in case series and associated to less pain, shorter hospital stay, faster return to normal activity, and more cosmetic incisions (6). A retrospective cohort study from the American College of Surgeons NSQUIP database found that, out of 512 patients with colovesical fistula $(85.5 \%$ diverticulitis), 152 (29.7\%) had a laparoscopic procedure. No bladder repair was needed in $36 \%$ of the patients. Interestingly, an open surgical approach was an independent predictor of postoperative morbidity [odds ratio (OR) 2.56, $95 \%$ confidence interval (CI), 1.35-4.84] in this study (7). In a large single-center series, 36 (40.5\%) patients were treated laparoscopically, with an overall $33.3 \%$ morbidity rate and no bladder leaks (8). Ureteral identification and protection is critical during minimally invasive pelvic surgery, and early recognition of injury can reduce postoperative morbidity associated with secondary procedures. Although there is no conclusive evidence supporting the effectiveness of nearinfrared fluorescence using ICG based on this single casereport, it has already been shown that intra-ureteral ICG allows to visualize the ureters during a variety of laparoscopic procedures for up to 6 hours (9-11). Moreover, this technique avoids the potential complications associated to double-J stenting by simply inserting the tip of an intra-ureteral catheter into the vesico-ureteric junction. On the other hand, the disadvantages of using ICG may be related to the need of direct ureteral injection which requires expertise and leads to additional surgical time (12). In our experience, close cooperation with the urologists allowed to safely manage our patient without significant prolongation of the operative time. We are convinced that the magnified laparoscopic vision enhanced by fluorescence allows a safe dissection of 
colovesical fistula and a safe colorectal anastomosis, and this may translate into a decrease frequency of bladder resection and defunctioning ileostomy.

\section{Acknowledgments}

This work was supported by AIRES (Associazione Italiana Ricerca ESofago).

Funding: None.

\section{Footnote}

Reporting Checklist: The authors have completed the CARE reporting checklist. Available at https://jovs.amegroups. com/article/view/10.21037/jovs-20-140/rc

Conflicts of Interest: All authors have completed the ICMJE uniform disclosure form (available at https://jovs. amegroups.com/article/view/10.21037/jovs-20-140/coif). The authors have no conflicts of interest to declare.

Ethical Statement: The authors are accountable for all aspects of the work in ensuring that questions related to the accuracy or integrity of any part in the work are appropriately investigated and resolved. All procedures performed in this study were in accordance with the ethical standards of the institutional research committee and with the Helsinki Declaration (as revised in 2013). Written informed consent was obtained from the patient for publication of this case report and accompanying images and videos. A copy of the written consent is available for review by the editorial office of this journal.

Open Access Statement: This is an Open Access article distributed in accordance with the Creative Commons Attribution-NonCommercial-NoDerivs 4.0 International License (CC BY-NC-ND 4.0), which permits the noncommercial replication and distribution of the article with the strict proviso that no changes or edits are made and the original work is properly cited (including links to both the formal publication through the relevant DOI and the license). See: https://creativecommons.org/licenses/by-nc-nd/4.0/.

\section{References}

1. Tomizawa K, Toda S, Tate T, et al. Laparoscopic surgery for colovesical fistula associated with sigmoid colon diverticulitis: a review of 39 cases. J Anus Rectum Colon
2019;3:36-42.

2. Kitaguchi D, Enomoto T, Ohara Y, et al. Laparoscopic surgery for diverticular colovesical fistula: single-center experience of 11 cases. BMC Res Notes 2020;13:177.

3. Burks FN, Santucci RA. Management of iatrogenic ureteral injury. Ther Adv Urol 2014;6:115-24.

4. Gagnier JJ, Kienle G, Altman DG, et al. The CARE guidelines: consensus-based clinical case reporting guideline development. J Med Case Rep 2013;7:223.

5. Klarenbeek BR, Bergamaschi R, Veenhof AA, et al. Laparoscopic versus open sigmoid resection for diverticular disease: follow-up assessment of the randomized control Sigma trial. Surg Endosc 2011;25:1121-6.

6. Cirocchi R, Arezzo A, Renzi C, et al. Is laparoscopic surgery the best treatment in fistulas complicating diverticular disease of the sigmoid colon? A systematic review. Int J Surg 2015;24:95-100.

7. Aydinli HH, Benlice C, Ozuner G, et al. Risk factors associated with postoperative morbidity in over 500 colovesical fistula patients undergoing colorectal surgery: a retrospective cohort study from ACS-NSQIP database. Int J Colorectal Dis 2017;32:469-74.

8. Dolejs SC, Penning AJ, Guzman MJ, et al. Perioperative Management of Patients with Colovesical Fistula. J Gastrointest Surg 2019;23:1867-73.

9. Siddighi S, Yune JJ, Hardesty J. Indocyanine green for intraoperative localization of ureter. Am J Obstet Gynecol 2014;211:436.e1-2.

10. Mandovra P, Kalikar V, Patankar RV. Real-Time Visualization of Ureters Using Indocyanine Green During Laparoscopic Surgeries: Can We Make Surgery Safer? Surg Innov 2019;26:464-8.

11. Kanabur P, Chai C, Taylor J. Use of Indocyanine Green for Intraoperative Ureteral Identification in Nonurologic Surgery. JAMA Surg 2020;155:520-1.

12. Slooter MD, Janssen A, Bemelman WA, et al. Currently available and experimental dyes for intraoperative nearinfrared fluorescence imaging of the ureters: a systematic review. Tech Coloproctol 2019;23:305-13.

doi: 10.21037/jovs-20-140

Cite this article as: Asti E, Bernardi D, Andreatta E, Conti A, Carmignani L, Bonavina L. Laparoscopic management of colovesical fistula secondary to sigmoid diverticulitis: case report and the role of intraoperative indocyanine-green fluorescence. J Vis Surg 2023;9:9. 